Rev. Elev. Méd. vét. Pays trop., 1979, 32 (4) : 379-389.

\title{
Le comportement sexuel du bélier Oudah
}

\author{
par Yves GAILLARD $(*)$
}

\begin{abstract}
RESUME
L'étude du comportement sexuel du bélier Oudah dans des conditions naturelles d'accouplement a été effectuée sur un petit troupeau à l'Université de Niamey, Niger, de janvier 1977 à juin 1979. Le comportement sexuel du bélier est décrit, ainsi que les chaînes de réactions comportementales entre mâle et femelle. Les fonctions des principaux éléments de comportement sont envisagées. Quelques données sur les performances reproductrices sont indiquées : maturité sexuelle, nombre de copulations par astrus, nombre journalier maximal de copulations, absence de variations annuelles.
\end{abstract}

\section{INTRODUCTION}

Le présent travail fait suite à un article consacré à la brebis Oudah (6), dans lequel cette race de moutons à poils du Sahel nigérien est présentée.

Il n'existe dans la littérature aucune étude sur la reproduction du bélier Oudah ni sur aucune race voisine africaine. Le comportement sexuel de différentes races de moutons à laine européens est par contre souvent décrit (BANKS (2) MATTNER et collab. (12), SAMBRAUS (16), FRASER (5), parmi les principaux). Nous essaierons au cours de cette étude de comparer certains aspects de la reproduction du Oudah avec ces moutons à laine.

\section{MATÉRIEL ET METHODES}

Nos animaux, ainsi que les conditions dans lesquelles ils sont élevés, sont décrits dans notre précédent article. Il en est de même pour les principales démarches expérimentales et les périodes d'observations effectuées.

En ce qui concerne plus particulièrement l'étude $d u$ comportement du bélier, nous avons utilisé essentiellement trois béliers adultes dès

(*) D-8069 Tegernbach. Waldweg 12, Allemagne. le début de nos observations ( 2,3 et 4 ans), et quatre autres béliers nés au laboratoire ont été suivis jusqu'à l'âge de 1,5 à 2,5 ans.

Ce travail présente principalement les résultats sur le comportement sexuel et les performances reproductrices au cours d'accouplements libres (béliers en permanence avec les brebis pendant toute la durée des chaleurs).

Différentes situations ont été réalisées pour observer l'influence de la hiérarchie sur le comportement sexuel: nombre variable de béliers d'âges différents ou identiques mis ensemble en présence des brebis, avec et sans femelle en œstrus.

\section{DESCRIPTION DU COMPORTEMENT SEXUEL DU BÉLIER OUDAH}

\section{Recherche et identification des brebis en aestrus}

Tout au long de ses périodes d'activité, le bélier effectue des contrôles génitaux et des contrôles d'urine des brebis, lui permettant ainsi de s'informer sur leur état oestrien.

\section{Contrôles génitaux}

Le bélier approche une brebis par l'arrière et flaire sa sphère ano-génitale. Si la femelle reste 
immobile, il termine parfois ce flairage par un frottage de la vulve avec le museau. Ces contrôles génitaux ne sont que rarement suivis d'un «Flehmen»: Le Flehmen, terme allemand utilisé généralement par les éthologistes, représente une mimique caractéristique des ruminants (et de nombreux autres ongulés); il consiste à relever très haut la lèvre supérieure, la tête tendue en avant au-dessus de l'horizontale. Ce Flehmen est principalement déclenché par l'odeur de l'urine.

\section{Contrôle d'urine}

La simple approche du mâle peut parfois déclencher une séquence de miction chez la brebis. Mais le plus souvent, ce ne sera qu'après toute une série de comportements typiques du bélier (réunis sous le terme de "chasses ») que la brebis urinera. Le bélier flaire alors le jet d'urine, le laissant couler légèrement sur son museau, les lèvres entr'ouvertes. Il donne parfois quelques coups de langue pour recueillir de l'urine dans la bouche. Chez les moutons à laine européens, le bélier semble au contraire éviter tout contact avec le jet d'urine qu'il flaire (16). Ce flairage de l'urine est pratiquement toujours suivi d'un Flehmen.

Après avoir effectué un contrôle d'urine sur une brebis non en cestrus, le bélier s'en désintéresse alors généralement.
La vue d'une brebis urinant à proximité incite aussi parfois le bélier à s'approcher et à contrôler cette brebis.

\section{Les « chasses 》}

La position de base est la posture "étirée " (Uberstrecken des auteurs allemands); à partir de cette posture, le bélier effectuera toute une série d'éléments de comportement, isolés ou groupés, d'intensité variable (ces différents éléments sont réunis sous le terme de nudging par les auteurs anglo-saxons).

Posture "étirée »: après un contrôle génital ou à l'approche d'une brebis, le bélier adopte cette position «étirée » : la tête et le cou sont tendus vers l'avant, légèrement au-dessus de l'horizontale, le nez pointé vers la brebis et le corps légèrement ramassé sur lui-même. Cette simple posture peut déclencher la miction de la brebis, mais en général elle se transforme en véritables "chasses" plus ou moins intenses.

«Chasses»: dans la forme la moins intense, le bélier, à partir de la position «étirée », a une brusque impulsion vers la brebis, accompagnée d'une rotation de la tête amenant le menton contre les flancs de la brebis. Ce mouvement est accompagné d'un mouvement alternatif rapide de la langue hors de la bouche (Zungenflippern des allemands) qui est souvent accompagné lui-même d'un bêlement guttural caractéristique.

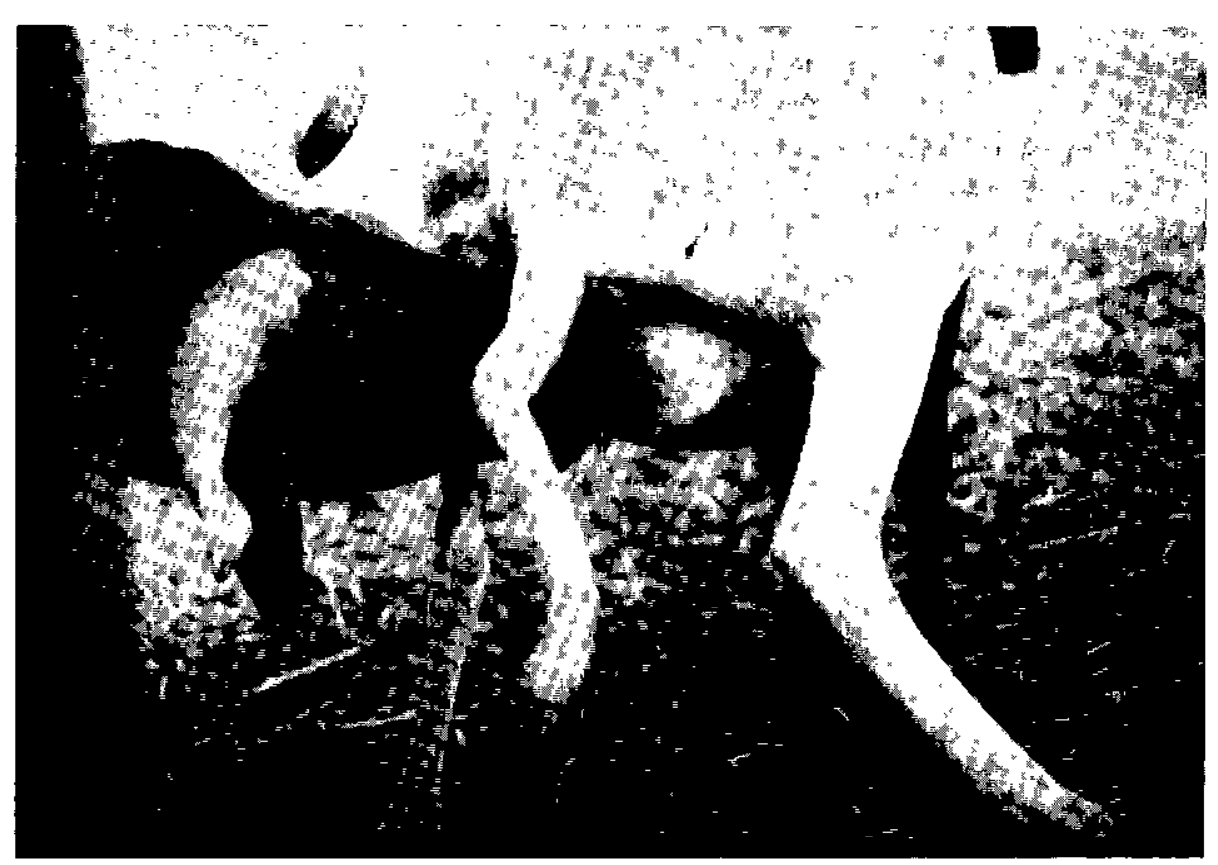

Photo $n^{0} 1 .-$ Contrôle d'urine. 
Fréquemment, surtout lorsque le bélier est proche de la brebis, il enchaîne ces mouvements avec des "coups de pattes" (Laufschlag des auteurs allemands); il lève brusquement un antérieur et tape le corps de la brebis avec le dessus du carpe, la bousculant parfois nettement. Dans la forme la plus intense, le bélier pourchasse la brebis à la course, en conservant la position étirée, et en effectuant dès qu'il la rattrape une rotation de la tête avec mouvements de la langue et bêlement guttural, plus rarement aussi des coups de pattes. Et ceci jusqu'à ce que la brebis urine, le bélier arrêtant alors ses poursuites pour contrôler l'urine. Nous verrons par la suite que les chasses intenses seront généralement réservées aux brebis en œst rus.

Il est intéressant de remarquer que les différents éléments des chasses font aussi partie du comportement agonistique entre béliers. La mictjon en réponse à un stress est une réaction physiologique normale chez la brebis. Il suffit d'effrayer brusquement une brebis pour déclencher une émission d'urine. La cérémonie du contrôle d'urine semble donc être une ritualisation à partir du comportement agonistique du bélier et de cette particularité de la brebis à uriner lorsqu'elle est effrayée.

\section{Parade sexuelle}

Lorsque la brebis entre dans sa période de réceptivité, le bélier va alterner ses "chasses" avec une série de comportements aboutissant au coït. Ces comportements se feront en réponse à ceux de la brebis et seront répétés de façon cyclique entre chaque coït.

\section{Grands cercles}

Les chasses décrites précédemment se retrouvent au cours de la parade mais sont plus intenses et accompagnées de coups de pattes plus actifs. A ce stade, les séquences de miction déclenchées chez la brebis sont en général négligées par le bélier qui continue ses chasses sans contrôler l'urine émise. Les animaux effectuent de grands cercles autour du troupeau.

\section{Petits cercles}

Au bout d'un certain temps, la brebis ne s'enfuira plus à la course devant les chasses du bélier. Elle ne se dérobe plus qu'en se retournant brusquement sur elle-même vers le bélier sous ses avances. Il s'ensuit une sorte de danse très caractéristique où les deux animaux tournent sur eux-mêmes dans une position parallèle tête-bêche, la brebis écartant brusquement son arrière-train à la moindre tentative du bélier pour s'orienter vers sa croupe.

\section{Tentative de monte de degré 1 (TM1)}

Le bélier, immobile à côté, perpendiculaire ou derrière la brebis, effectue les éléments des chasses décrits plus haut, mais sans déplacements et avec de violents coups de patte.

\section{Tentative de monte de degré 2 (TM2)}

Immobile derrière ou perpendiculaire à la brebis, le bélier lui pose le menton sur la croupe ou le dos et a une brusque impulsion vers l'avant accompagnée de mouvements de la langue et de bêlements gutturaux, ainsi que des coups de patte contre les flancs ou entre les cuisses de la brebis. Pendant ces mouvements, il tend à s'orienter vers l'arrière de la brebis. Ceci représente un mélange entre les TM1 et les TM3.

\section{Tentative de monte de degré 3 (TM3)}

Le bélier, derrière la brebis ou parallèle en retrait, pose le menton sur sa croupe et effectue une forte pression du menton sur la croupe; en même temps il fait un profond mouvement du bassin vers l'avant accompagné d'une forte érection. C'est une très nette amorce de monte, les membres antérieurs s'élevant même parfois légèrement $\mathrm{du}$ sol.

Pendant ces TM, les coups de patte prennent une importance considérable et sont de trois types :

- A l'aide d'un antérieur relevé à l'horizontale et raidi, le bélier tapote la brebis sous le ventre ou entre les cuisses;

- Véritables coups avec le dessus du carpe contre les flancs ou entre les cuisses de la femelle, la bousculant nettement;

- Simple amorce de coup de patte conduisant à un grattage du sol avec le sabot, le plus souvent entre ou contre les pattes arrières de la brebis. Cela surtout lorsque le bélier commence à montrer des signes d'épuisement. 


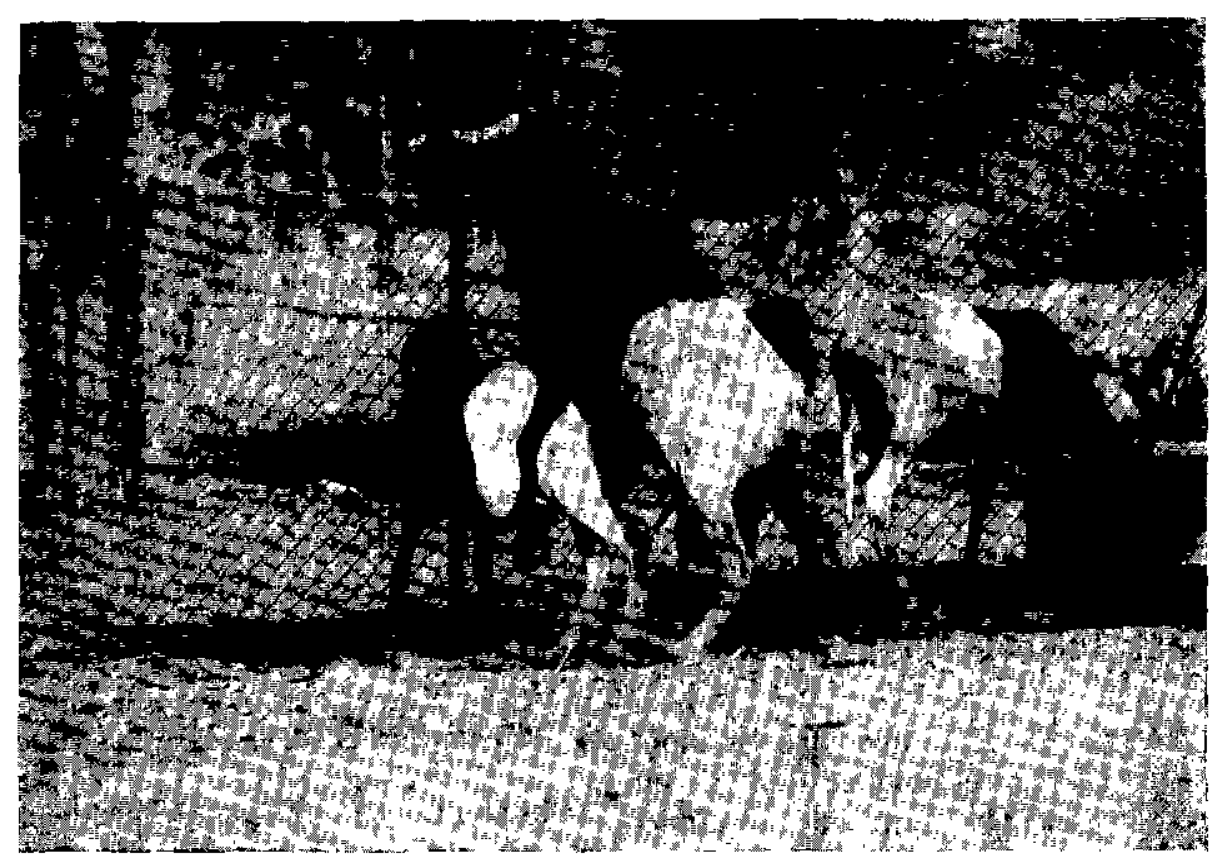

Photo $\pi^{\circ} 2$. - Monte avec intromission.

\section{Montes sans copulation (MsC)}

Avant chaque coït, le bélier effectue un certain nombre de montes avec ou sans intromission du pénis, accompagnées ou non de mouvements alternatifs du bassin, mais sans éjaculation.

Même lorsque la brebis reste parfaitement immobile, le bélier effectuera quelques TM et $\mathrm{MsC}$ avant le véritable coït.

\section{Coït}

Après les différents comportements décrits plus haut, l'une des montes avec intromission du pénis et accompagnée de mouvements du bassin (de trois à quinze) aboutit à une poussée plus profonde vers l'avant, cette poussée traduisant seule l'éjaculation. Pendant le coït, le poitrail du bélier repose fortement sur la croupe de la brebis, sa tête et son cou sont dressés en l'air et il enserre le corps de la brebis avec ses membres antérieurs, l'articulation du genou épousant étroitement le grasset de la brebis, le reste de l'avant bras étant appliqué dans le sillon entre les tubercules ischiadique et sacré de la brebis. Chez tous les autres moutons (moutons à laine européens (16), mouflons propres observations) le mâle enserre la brebis en avant des tubercules sacrés (photo $n^{\circ} 2$ ).

\section{Comportement post-copulatoire}

Après une copulation, le mâle redescend et reste un instant immobile tête baissée à côté de la brebis. Puis il broute ou la suit tranquillement. Après quelques minutes ou plus, il effectue à nouveau quelques contrôles génitaux et d'urine, aussi bien sur la brebis en cstrus que sur d'autres voisines, puis il reprend la parade sexuelle proprement dite.

\section{CHAINES DE RÉACTIONS. LES DÉCLENCHEURS ET LEURS FONCTIONS}

Aussi bien dans les interactions entre le bélier et la brebis non en cestrus qu'avec la brebis en œstrus, les chaînes de réactions mises en jeu sont particulièrement bien orientées et stéréotypées pour des mammifères supérieurs. Nous essayons de représenter ci-après ces chaînes de réactions.
Légende :
$\Rightarrow$ fréquent
$\rightarrow$ moins fréquent
(*) ce niveau peut être répété plusieurs fois
$\rightarrow$ rare
(1) ce niveau peut man- quer 
Chaîne de réactions entre bélier et brebis en oestrus

Q

Fonmes, mouvements, odeur

fuit

urine

grand $\mathrm{B}$ cercles
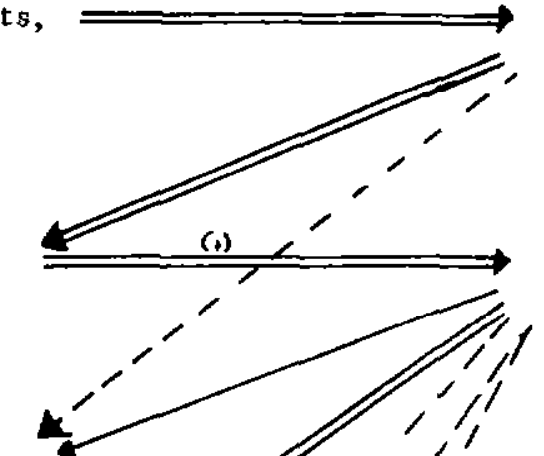

elhasses intenses

s'approche, contrôle

gënital, chasses

lëgères dẹrobades

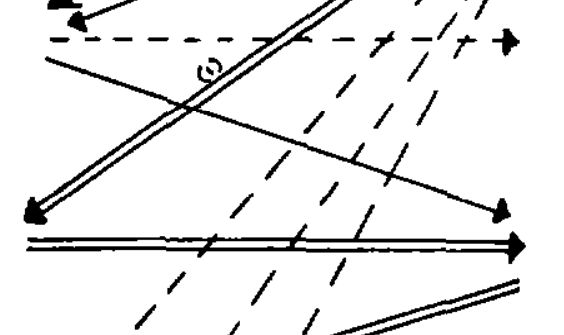

petits cercles

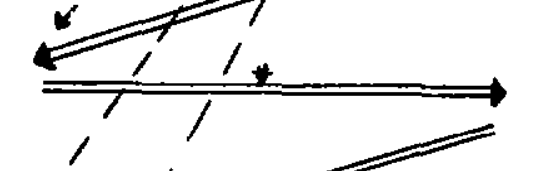

chasses, TM

reste immobile retourne la tête vers le bêlier

reste immobile

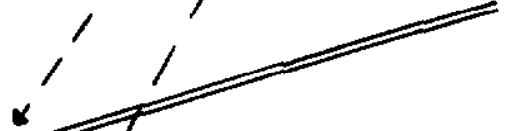

chasses, TM, MsC

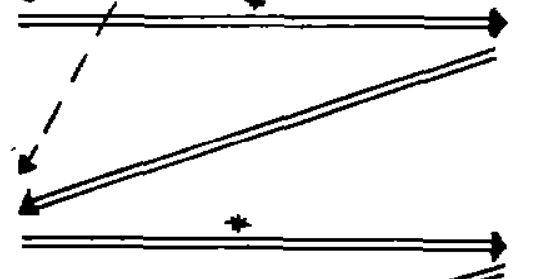

$\mathrm{TM}, \mathrm{MsC}$

monte avec intromission et mouvements du bassin

position typique, base de la queue arquée

flaire les flancs ou le pénis du bélier.<smiles>II(I)[Ge](I)(I)I(I)[Ge](I)(I)I</smiles>

reste un instant immobile et reprend ou non sa cour. 


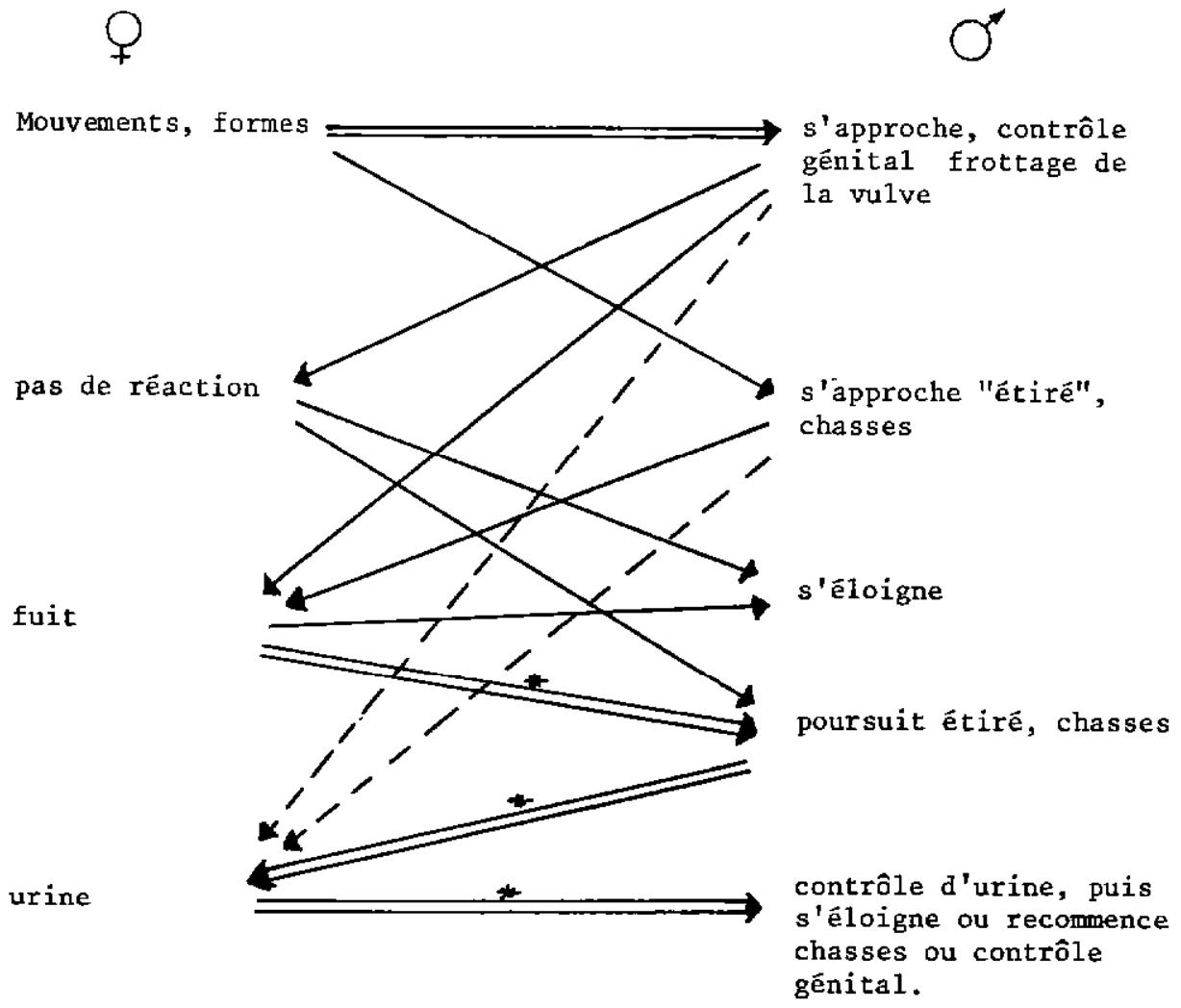

Certains niveaux de cette chaîne de réactions peuvent manquer ou au contraire être répétés un nombre variable de fois, suivant l'état de réceptivité de la brebis et l'état sexuel du bélier.

\section{Les déclencheurs et leurs fonctions}

- Reconnaissance de l'état cestrien d'une brebis par le bélier : C'est le rôle principal des contrôles génitaux et d'urine.

- Accélération de la venue de l'œstrus et de l'ovulation: Le frottage fréquent de la vulve après un contrôle génital, ainsi que la présence et le comportement global du bélier accélèrent la venue de l'œstrus et le déclenchement de l'ovulation, ce qui entraîne un raccourcissement de la durée de l'œstrus. Cela a été mis en évidence chez différents ongulés (4), chez la vache (7) et chez le mouton $(13,15,1,18)$.

- Test d'immobilisation, synchronisation des partenaires : Les chasses effectuées envers une brebis en œstrus, les TM et les MsC servent à tester la réceptivité de la brebis, qui se traduit par son immobilité aux avances du bélier.
- Amélioration de la libido et des qualités du sperme : Les chasses, $\mathrm{TM}$ et $\mathrm{MsC}$, outre leur rôle de test d'immobilisation, ont une influence très importante sur les paramètres physiologiques nécessaires à la reproduction du bélier : SIGNORET (17) a montré chez les bovins que la poursuite de la femelle par le mâle augmente sa libido sans influencer les qualités du sperme ; les montes sans copulation par contre améliorent très nettement les qualités du sperme : l'effet sur la concentration en spermatozoïdes par exemple est la suivante :

\section{Comportement}

Nombre de spermatozoïdes par éjaculat

Aucune MsC prélimi-

naire

Après une $\mathrm{MsC}$

Après deux $\mathrm{MsC}$ en $5 \mathrm{mn}$

$$
\begin{aligned}
& 3 \times 10^{9} \\
& 5,2 \times 10^{9} \\
& 8 \times 10^{9}
\end{aligned}
$$

Il existerait donc deux mécanismes distincts, l'un augmentant la libido, l'autre la qualité du sperme. 


\section{ÉTUDE QUANTITATIVE}

\section{Eléments de comportement}

\section{Contrôles génitaux}

Le nombre de contrôles génitaux effectués par jour par un bélier est assez constant pour un troupeau de dix à quatorze brebis, et ils sont distribués à peu près également entre les brebis. Ce nombre s'élève nettement lorsqu'une brebis est en œstrus, environ la moitié des contrôles lui étant alors réservés.

Pour 11 jours d'observation sans brebis en cstrus dans le troupeau, et en utilisant deux béliers différents, nous avons observé entre quatre-vingt-six et cent trente-trois contrôles génitaux par jour pour un bélier. La moyenne journalière est de 105,2 $\pm 7,4$.

Avec au moins une brebis en astrus dans le troupeau, nous trouvons une valeur moyenne de $155,2 \pm 6,2$ (15 jours d'observation) (Graphique 1).

\section{Contrôles d'urine (CU)}

Nous avons vu que les contrôles d'urine sont presque toujours liés à un élément de comportement des «chasses». Aussi nous donnons ci-après le pourcentage des contrôles d'urine succédant à ces différents éléments de comportement, en séparant les jours avec ou sans brebis en cestrus :
Avec 우

p. 100 des contrôles génitaux suivis d'un $\mathrm{CU}$ 9,2 p. $100 \quad 10 \quad$ p. 100

p. 100 des «chasses» suivies d'un CU 6,3 p. $100 \quad 25,9$ p. 100

La différence observée entre les jours avec ou sans brebis en cestrus montre bien les fonctions différentes que possèdent les chasses suivant qu'elles s'adressent à une femelle en cestrus ou non.

Chez les races à laine européennes, les béliers contrôlent surtout l'urine des brebis en astrus $(2,16)$.

\section{Chasses}

Pour pouvoir suivre l'évolution quantitative de ces éléments, nous avons considéré trois degrés différents :

- «chasses » isolées, sans déplacement ;

- "chasses" isolées avec déplacement ;

- série de chasses (traduisant une poursuite avec plus de deux "chasses» successives).

Le nombre de chasses augmente de façon importante en présence d'une brebis en cestrus et elles sont adressées pour près de 70 p. 100 à cette brebis. Sans brebis en castrus, les chasses sont distribuées à peu près également entre les brebis.

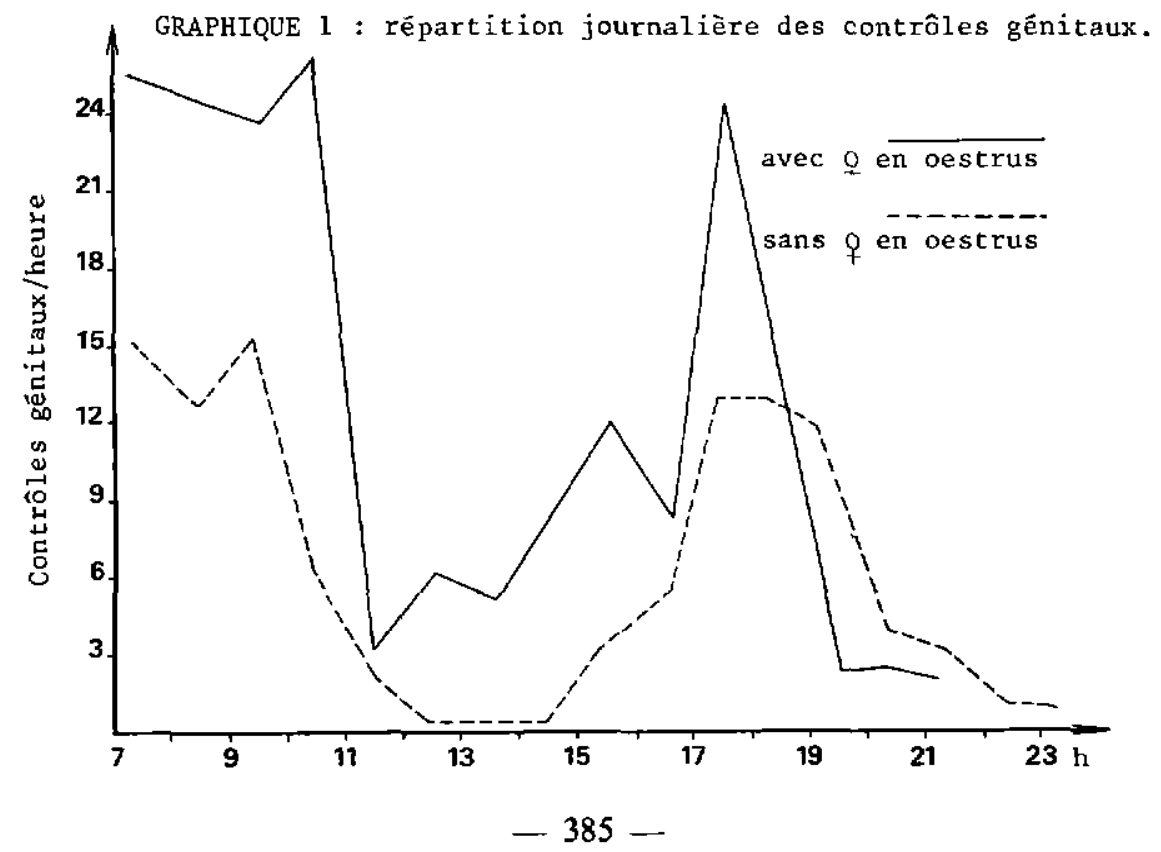




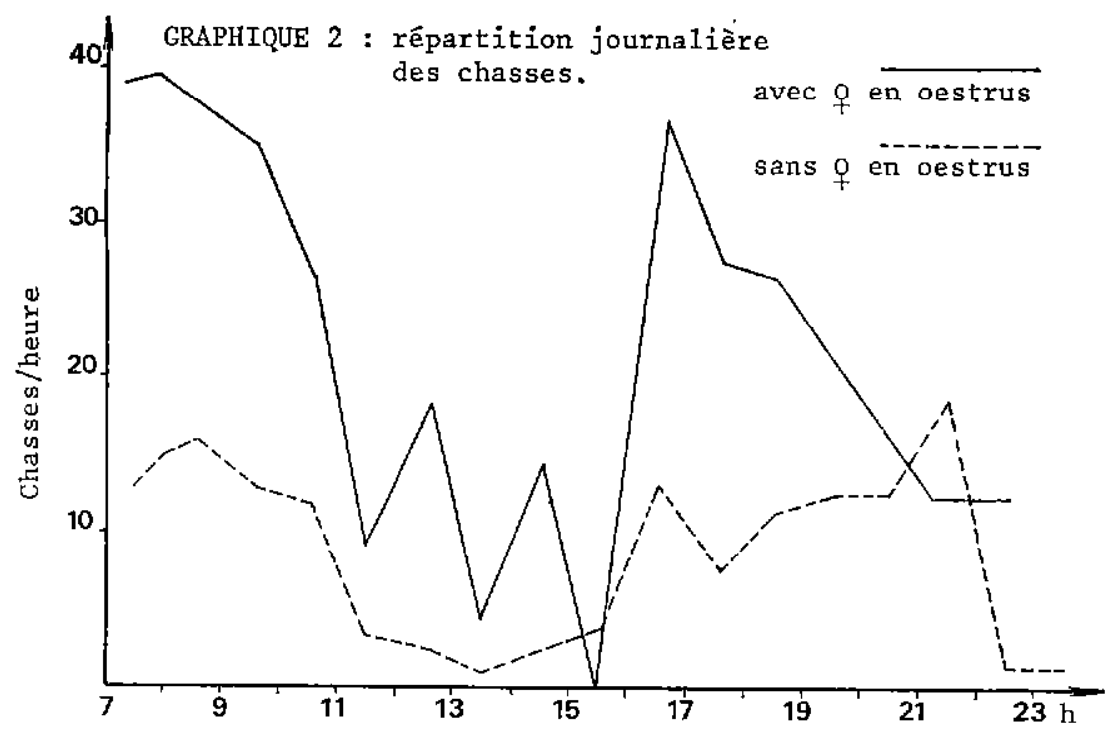

On remarque que les «grands cercles 》 de la parade sexuelle comportent de très longues séries de ces «chasses » (Graphique 2).

\section{Tentatives de monte et montes sans copulation}

Avant chaque copulation, le nombre de TM effectués par un bélier est très variable: de quatre à vingt. Le nombre de $\mathrm{MsC}$ est par contre beaucoup plus stable et nous avons calculé une moyenne de $3,9 \pm 1,0 \mathrm{MsC}$ précédant chaque coït (résultats calculés sur deux cent quarantetrois copulations de deux béliers différents).

Chez différentes races de béliers à laine européens, ce nombre est nettement inférieur :

- SAMBRAUS (16) indique que sur mille soixante-quatre copulations de quatorze béliers différents il obtient une moyenne de 1,4 MsC par coït.

- EDNEY et collab. (3) précisent que ce nombre varie suivant l'âge et le rang hiérarchique du bélier, avec des extrêmes de 2,7 à 13,9.

- MATTNER et collab. (12) font les mêmes remarques et trouvent une valeur moyenne de 1,61 MsC par coĭt.

\section{Durée du couple}

Le bélier détecte 1 à 3 jours à l'avance une brebis qui va être en cestrus. Il la poursuivra de plus en plus régulièrement dans les quelques heures précédant le premier coït. Puis le couple sera remarquablement stable pendant la pre- mière journée de l'æstrus, le bélier ne quittant que rarement la brebis. Le deuxième jour par contre, il la quittera de plus en plus souvent et longtemps pour s'en désintéresser même parfois complètement avant la fin de l'œstrus.

Deux phénomènes feront varier de façon importante la durée du couple: la présence d'autres béliers renforce et rallonge la durée du couple formé alors par le bélier dominant ; au contraire, lorsqu'une seconde brebis entre en astrus, le bélier abandonne très vite la première pour former un nouveau couple avec la seconde.

\section{Quelques caractéristiques des performances repro- ductrices}

Nombre de coïts, intervalle entre les copulations

Le nombre de copulations effectuées par un bélier au cours d'un cstrus est assez variable suivant la brebis et l'œstrus envisagés, mais il dépendra surtout du bélier. Si au début de l'œstrus c'est en général la brebis qui limite la fréquence des copulations, très vite le bélier va perdre de l'intérêt pour cette brebis et l'intervalle entre les copulations successives s'allongera. Il en résulte des valeurs moyennes peu significatives, si ce n'est pour donner un ordre de grandeur : dix à vingt-cinq copulations par œstrus (en alternant des béliers toutes les 2 ou $3 \mathrm{~h}$ au cours d'un œstrus, nous avons obtenu sur deux brebis trente-cinq et quarante copulations acceptées en $48 \mathrm{~h}$ ). 
Lorsqu'il y a plusieurs brebis en estrus dans la même journée, le mâle effectuera un maximum de vingt à trente copulations par jour.

Chez quelques races européennes à laine, des valeurs maximales moyennes journalières de seize à vingt-cinq copulations sont données $(8,12,16)$.

\section{Fécondité}

Bien que le nombre de nos animaux ne permette pas une étude de la fécondité, nous ferons la remarque suivante : dans quatre cas, il y a eu trois à quatre brebis à la fois en cstrus, avec un seul bélier dans le troupeau. Or à chaque fois, moins de 50 p. 100 des brebis furent fécondées bien que toutes aient été couvertes à plusieurs reprises. En dehors de ces cas, sur vingtdeux accouplements contrôlés, il y a eu 91,6 p. 100 de fécondations.

\section{Maturité sexuelle}

Les éleveurs nigériens considèrent les béliers aptes à la reproduction entre 8 et 9 mois.

Nous avons testé quatre jeunes béliers et obtenu la première copulation fécondante entre 8 et 12 mois suivant le bélier.

Pour différentes races européennes, FRASER (5) donne 5 à 9 mois, HAFEZ et HAIRE dans VAISSAIRE (19) donnent 3,8 à 6 mois comme âge de maturité sexuelle.

\section{Variations annuelles}

Le bélier Oudah, de même que la brebis, est apte à se reproduire toute l'année et ne montre aucun signe de diminution de sa libido à une période donnée.

Chez les moutons européens, quelques travaux semblent montrer que la saisonnalité de la reproduction n'est pas seulement liée à un anœstrus saisonnier des brebis, mais que la libido et la puissance sexuelle du bélier sont liées aussi aux variations photopériodiques $(9,14,10)$.

\section{INFLUENCE DE LA HIÊRARCHIE}

Chez le bẻlier Oudah, l'agressivité est étroitement liée à la sexualité. L'agressivité entre les béliers augmente de façon très importante dès qu'une brebis est en œstrus.
En mettant en présence des béliers d'âges différents avec les brebis, nous avons fait les observations suivantes:

- Deux béliers de haut rang hiérarchique (4 à 6 ans) se combattent très violemment dès qu'ils sont mis en présence, qu'il y ait ou non une brebis en œstrus dans le troupeau;

- Des béliers de rangs hiérarchiques très différents (jeunes de 1 à 2 ans avec béliers de 4 à 6 ans) restent à peu près indifférents tant qu'il n'y a pas de brebis en œstrus. En présence de brebis en œstrus, par contre, le bélier dominant devient très agressif et charge violemment dès qu'un inférieur s'approche de la brebis en cestrus ou de lui-même.

- Seule la présence de plusieurs brebis en astrus à la fois laisse une chance de s'accoupler aux béliers de rang inférieur.

Chez les moutons à laine européens, la hiérarchie joue aussi un rôle important dans la reproduction : une corrélation directe entre le rang hiérarchique et le nombre de copulations effectuées est toujours observée, le bélier dominant se réservant le maximum de copulations (16, 2, 12).

MARINCOWITZ (11) a montré que la libido et la puissance sexuelle sont directement liées au rang hiérarchique potentiel du bélier : après avoir établi le rang hiérarchique de cinquante-six béliers, chacun d'entre eux fut laissé avec un troupeau de deux cents brebis pour une saison de monte de 2 mois.

- Les béliers dominants ont couvert pratiquement toutes les brebis et leur libido a persisté très longtemps.

- Les béliers de rang hiérarchique moyen ont couvert au début autant de brebis que les dominants, mais ils ont perdu rapidement leur libido.

- Les béliers de rang inférieur n'ont dès le début qu'une faible libido et n'ont couvert que peu ou même pas du tout de brebis.

Or il n'a pu être trouvé aucune corrélation entre le poids ou d'autres caractères morphologiques entre les béliers et leur rang hiérarchique, si ce n'est l'existence d'un plus grand nombre de replis de peau sur le cou chez les inférieurs. Le rang hiérarchique potentiel semble donc être fixé héréditairement, en corrélation avec la libido et la puissance sexuelle. 


\section{CONCLUSION}

Le comportement sexuel du bélier Oudah est très voisin de celui de nos moutons à laine européens. Cependant, quelques différences très nettes sont à noter : une plus grande variété d'éléments de comportements et une position d'enserrement lors des montes tout à fait caractéristique.

Au niveau des chaînes de réactions du comportement, la différence est encore plus nette : ces chaînes de réaction sont beaucoup mieux ritualisées et stéréotypées chez le Oudah, très simplifiées et raccourcies au contraire chez les races domestiques européennes.
$\mathrm{Au}$ niveau des capacités reproductrices, nous remarquons une maturité sexuelle plus tardive chez le Oudah, ainsi que l'absence de variation saisonnière dans la reproduction.

\section{REMERCIEMENTS}

L'auteur remercie vivement l'Université de Niamey, et tout particulièrement Monsieur BA, Recteur de l'Université, Monsieur FOULANI, Directeur de l'Ecole des Sciences et Monsieur YENIKOYE, Chef du service de Biologie, qui m'ont permis et facilité la réalisation de ce travail.

\section{ZUSAMMENFASSUNG}

Von Januar 1977 bis Juni 1979 wurde an einer kleinen Herde von OudahSchafen das Sexualverhalten unter natürlichen Bedingungen, an der Université Niamey, Niger, studiert. Beschrieben werden das Sexualverhalten des Widders, sowie die Reaktionsketten zwischen Weibchen und Männchen. Die Funktionen der wichtigsten Verhaltensweisen werden erwogern. Einige Daten zur sexuellen Leistung des Widders werden angegeben: Geschlechtsreife, Zahl der Kopulationen pro Oestrus, höchste Kopulationanzahl pro Tag, das Fehlen einer Fortpflanzungssaison.

\section{SUMMARY}

\section{Sexual behaviour of Oudah ram}

From January 1977 until June 1979 I was studying the sexual behaviour under natural condition on a small flock of Oudah-sheep at the University of Niamey, Niger. The sexual behaviour of the ram is described, as well as the series of reactions between females und males. The functions of the most important behaviours patterns are considered. There are some dates concerning the sexual capacity of the ram : puberty, number of matings per cestrus, highest mating rate per day, the lack of a breed season.

\section{RESUMEN}

\section{El comportamiento sexal del morueco Oudah}

Se efectuó el estudio del comportamiento sexual del morueco Oudah en las condiciones naturales de apareamiento en un pequeño rebaño en la Universidad de Niamey, Niger, de enero 1977 a junio 1979. Se describen el comportamiento sexual del morueco así como el desarrollo de las reacciones del comportamiento entre el macho y la hembra. Las funciones de los principales elementos de comportamiento son examinadas. Se indican algunos datos sobre la capacidad de reproducción : maturidad sexual, número de cópulas por estrus, número diario maximo de cópulas, ausencia de variaciones anuales.

\section{BIBLIOGRAPHIE}

1. BADINAND (F.). Synchronisation des chaleurs chez les petits ruminants. Econ. Méd. anim., 1970, $11(6)$ : 333-346.

2. BANKS (E.). Some aspects of sexual behaviour in domestic sheep, Ovis aries. Behaviour, 1964, 23 : 249-279.

3. EDNEY (T. N.), KILGOUR (K.), BREMMER (K.). Sexual behaviour and reproductive performance of ewe lambs at and after puberty. J. agric. Sci., 1978, $90(2)$ : 83-91.
4. FRASER (A. F.). Reproductive behaviour in Ongulates. London-New York. Academic Press 1968.

5. FRASER (A. F.). Farm animal behaviour, London, Baillère Tindall, 1974.

6. GAILLARD (Y.). Caractéristiques de la reproduction de la brebis Oudah. Rev. Elev. Méd, vét. Pays trop., 1979, 32 (3) : 283-288.

7. HINTNAUS (J.). Reflex-induced oestrus in cattle with weak or no signs of heat. Vet. Med. Praha, $1965,10: 69-76$. 
8. LAMBOURNE (L. J.). Mating behaviour, Proc. Ruakura Farmer's Conf. Week, 1956.

9. LEES (J. C.). Seasonal variations in the breeding activity of rams. Nature, 1965, $207: 221-222$.

10. LINCOLN (G. A.), DAVIDSON (W.). Relationships between sexual and aggressive behaviour and pituitary and testicular activity during the seasonal sexual cycle of rams, and the influence of photoperiode. J. Reprod. Fert., 1976, 49 : 267-276.

11. MARINCOWITZ (G.), PRETORIUS, HERBST. Dominance in sexual behaviour and mating dexterity in merino rams. $S$. Afr. agric. Sci., 1966, $9: 971-$ 980.

12. MATTNER (P. E.), BRADEN, TURNBULL. Studies in a flock mating of sheep : Mating behaviour. Austr. J. exp. Agric. anim. Husb., 1967, 7 : 103-109.

13. PARSON (S. D.), HUNTER (G. L.). Effect on the ram on duration of cestrus in ewe. J. Reprod. Fert., 1967, $14: 61-70$.

14. PEPELKO (W. E.), CLEGG (M. J.). Influence of season of the year upon patterns of sexual behaviour in male sheep. J. anim. Sci., 1965, $24: 633-637$.
15. PRUD'HON (M.), DENOY (I.). Effets de l'introduction de béliers vasectomisés dans un troupeau de mérinos d'Arles, 15 jours avant le début de la lutte de printemps, sur l'apparition des cestrus, la fréquence des erreurs de détection des ruts et la fertilité des brebis. Annls. Zootech., 1969, 18 : 95-106.

16. SAMBRAUS (H. H.). Das Sexualverhalten der domestizierten einheimischen Wiederkäuer. $Z$. Tierpsych., 1973, Beiheft 12.

17. SIGNORET (J. P.). Effets de l'influence de divers éléments du comportement sexuel du taureau sur les caractéristiques du sperme. Annls Zootech., 1962, 11 (2) : 93-101.

18. SIGNORET (J. P.). Influence of the presence of rams on the luteinizing hormone surge after cestradiol benzoate injection in ovariectomized ewes. $J$. Endocrinol., 1975, 64 : 589-590.

19. VAISSAIRE (J. P.). Sexualité et reproduction des mammifères domestiques et de laboratoire. Paris, Maloine, 1977. 